\title{
ANGUILLES ESTUARIENNES ET FLUVIALES : APPORTS DE L'OTOLITHOMÉTRIE.
}

\author{
Béatrice MOUNAIX, G. FONTENELLE
}

\begin{abstract}
Unité Halieutique, Département Environnement et Exploitation des Ressources Naturelles, Ecole Nationale Supérieure Agronomique, 65 rue St Brieuc, 35042 RENNES Cedex, France.
\end{abstract}

\section{RÉSUMÉ}

Des prélèvements d'anguilles (Anguilla anguilla) femelles ont été effectués dans les bassins versants de la Vilaine $(n=569)$ et de la Loire $(n=221)$, en zone fluviale et dans les deux estuaires. Les otolithes ont été poncés suivant le plan sagittal et le patron d'agencement des lignes d'arrêt de croissance (LAC) révélées par coloration au bleu de toluidine a été étudié avec une attention particulière pour l'origine fluviale ou estuarienne des échantillons. Pour une même classe de taille des poissons, les otolithes des anguilles fluviales présentaient jusqu'à deux fois plus de lignes d'arrêt de croissance que ceux des anguilles estuariennes. L'observation de la microstructure des LAC en microscopie électronique à balayage a conduit à distinguer deux catégories de LAC : des LAC composites et larges $(>10 \mu \mathrm{m})$, préalablement validées comme les annuli saisonniers, et des LAC simples et étroites $(<5 \mu \mathrm{m})$ représentatives de zones non saisonnières. Tous les otolithes des anguilles fluviales présentaient les deux catégories de marques. Parmi les otolithes des anguilles estuariennes, seuls $5 \%$ de l'échantillon de la Vilaine et $8 \%$ de celui de la Loire présentaient des LAC simples.

La zonation de l'otolithe, interprétée par rapport à la croissance des poissons, décrit deux écotypes dans la population d'anguilles de la Vilaine : un écotype fluvial dont la croissance est perturbée et ralentie, et un écotype estuarien dont la croissance est plus régulière. Cette partition de la population est cohérente avec les caractéristiques environnementales des deux milieux. Elle pourrait par ailleurs constituer un indice de l'impact des aménagements des bassins versants sur la croissance, la durée du cycle biologique et la dynamique des populations d'anguilles. II apparal̂t alors nécessaire de considérer séparément les sous-unités estuariennes et fluviales des stocks d'anguilles des bassins versants de la Vilaine et de la Loire, en particulier pour étudier la diminution des abondances.

Mots-clés : anguille, Anguilla anguilla, croissance, identification des stocks, otolithe.

\section{FLUVIAL AND ESTUARINE EELS : CONTRIBUTIONS OF OTOLITHOMETRY.}

\section{ABSTRACT}

Female eels (Anguilla anguilla) have been sampled in two water basins, the Vilaine river $(n=569)$ and the Loire river $(n=221)$, both in estuaries and fluvial streams. Otoliths have been ground on the sagittal plane and pattern of growth zones (LAC, stained by toluidine blue) was described with a special attention to the sampling site, fluvial or estuarine. For fish of the same length class, LAC were up to twice as numerous in fluvial eel otoliths than in estuarine ones. The microstructure of LAC was observed with a scanning electronic microscope and two types of LAC were distinguished : composite and large $(>10 \mu \mathrm{m})$ LAC that have been validated as seasonal annuli, and simple and narrow $(<5 \mu \mathrm{m})$ LAC which correspond to non-seasonal zones. All the otoliths of fluvial eels displayed both types of LAC. Among the otoliths of estuarine eels, only $5 \%$ of the Vilaine sample and $8 \%$ of the Loire one displayed simple LAC. 
Otolith zonation, related to fish growth, describes two ecotypes in eel populations : a fluvial ecotype which growth is disturbed and slowed, and an estuarine ecotype which growth is more regular. This partition of the population is coherent with environmental characteristics of both biotopes. It could constitute an index of water basin management on growth, duration of life cycle and population parameters of eels stocks. Therefore, it seems necessary to distinguish fluvial and estuarine sub-units of eel populations in both water basins, especially when studying the decrease of abundances.

Key-words : eel, Anguilla anguilla, growth, stock identification, otolith.

\section{INTRODUCTION}

Dans la majorité des bassins versants bretons, on observe une diminution marquée des densités d'anguilles, Anguilla anguilla L. (LEGAULT et PORCHER, 1990). Le bassin versant constitue l'unité de gestion appropriée pour cette espèce (GASCUEL, 1987 ; GASCUEL et FONTENELLE, 1989), mais il apparaît nécessaire de distinguer plusieurs fractions au sein de la population d'anguilles d'un même bassin.

En effet, l'Anguille est un poisson ubiquiste : elle colonise l'ensemble des compartiments du bassin versant, de l'estuaire aux zones fluviales situées en amont. La variété des sites colonisés entraîne une forte variabilité des structures démographiques (APRAHAMIAN, 1988), du sexe-ratio et de la croissance (VOLLESTAD, 1989). En outre, l'anguille est un poisson migrateur amphihalin : sa capacité de déplacement au sein d'un bassin versant (pour le coloniser ou retourner en mer) est un paramètre déterminant de la durée du cycle biologique (HANSEN et EVERSOLE, 1984 ; HARO et KRUEGER, 1991). L'isolement, artificiel ou non, de fractions estuariennes et fluviales au sein d'une population d'anguilles est donc à même de conditionner l'efficacité de la colonisation des zones amont du bassin et d'engendrer des caractéristiques biologiques différentes.

L'âge et la croissance des poissons peuvent être interprétés a posteriori à partir des marques de croissance observées dans les otolithes (CAMPANA et NEILSON, 1985 ; CASSELMAN, 1990). L'étude présentée fait suite à la mise au point d'une méthode validée de détermination de l'âge des anguilles (MOUNAIX, 1992) et sera complétée par l'analyse chimique des otolithes, en cours de réalisation : elle a pour but de décrire les patrons de croissance des otolithes des anguilles dans deux bassins versants bretons et d'interpréter la croissance des poissons en fonction de leur biotope. L'attention a été dirigée sur les Lignes d'Arrêt de Croissance (LAC), telles que définies dans BAGLINIERE et al., 1992 : elles correspondent à un changement de structure de l'otolithe et sont colorées par le bleu de toluidine. Elles sont localisées à la périphérie externe des zones hyalines (MOUNAIX, 1992).

\section{MATÉRIEL ET MÉTHODES}

\section{Le site d'étude}

L'étude a été menée en Bretagne, dans le bassin versant de la Vilaine et en Loire maritime (en aval de St Florent). Le bassin versant de la Vilaine (10 $800 \mathrm{~km}^{2}$ ) comporte un barrage estuarien, non équipé de passe et infranchissable pour les anguilles en dehors des périodes d'ouverture de l'écluse de navigation. Le bassin versant de la Loire $\left(120000 \mathrm{~km}^{2}\right)$ ne possède pas de barrage estuarien.

Deux sites d'échantillonnage ont été choisis dans chaque bassin : une pêcherie commerciale en estuaire, et un site fluvial distant de $100 \mathrm{~km}$ du premier. Les deux sites fluviaux différaient fortement : en Vilaine, les anguilles ont été capturées dans un affluent large de 2 à $3 \mathrm{~m}$ pour une profondeur comprise entre $15 \mathrm{~cm}$ et $1 \mathrm{~m}$. En Loire, les anguilles provenaient d'une zone du cours principal large $(10 \mathrm{~m})$ et profonde $(2 \mathrm{à} 6 \mathrm{~m})$.

\section{Collecte des données}

Seules les femelles ont été considérées dans le cadre de cette étude, pour éliminer la variation de croissance liée au sexe. Elles représentent généralement la majorité des captures et sont considérées comme responsables de la colonisation des zones amont. 
En Vilaine, 289 anguilles ont été chalutées en zone estuarienne et 280 anguilles ont été capturées par pêche électrique en zone fluviale. Différents prélèvements ont été collectés entre juin 1989 et juin 1991, cumulés dans le cadre de cette présentation. En Loire, deux lots (91 anguilles estuariennes et 130 anguilles fluviales) ont été échantillonnées chez les mareyeurs en octobre 1990.

Chaque poisson a été mesuré (longueur totale : LT, au $\mathrm{mm}$ près) et pesé (poids total : PT, au g près). Les gammes de taille et de poids similaires ont permis les comparaisons entre les différents lots. Les otolithes (Sagitta) ont été extraits et mesurés au micron près selon le plus grand diamètre (axe antéro-postérieur, diamètre maximal : DO) à l'aide d'un système d'analyse d'image adapté (système MAAO* Olympus). Après inclusion dans de la résine dentaire (Promodentaire ${ }^{T M}$ ), les otolithes ont été poncés sur du papier abrasif et polis à l'aide de poudre d'alumine. Cette procédure a pour but de dégager le nucleus de l'otolithe. La décalcification de la surface polie à l'aide d'EDTA ( $1 \%$ durant $5 \mathrm{mn}$ ) suivie de sa coloration au bleu de toluidine $(5 \%$ durant $5 \mathrm{mn}$ ) révèle les Lignes d'Arrêt de Croissance (LAC). Le patron d'agencement de ces lignes a été observé à la loupe binoculaire (x 7,5 à x 64). Le système d'analyse d'image a permis de mesurer le diamètre du nucleus, le nombre de LAC et leur diamètre (selon l'axe antéropostérieur de l'otolithe).

Après lavage de la coloration à l'alcool à $70^{\circ}$, un sous-échantillon d'otolithes a été observé en microscopie électronique à balayage (MEB, JEOL JSM 6400) pour décrire la microstructure des LAC (grossissement $\times 1000$ à $\times 2000$ ).

\section{Test statistique des différences}

Les corrélations ont été estimées à l'aide du coefficient de corrélation par rang de Spearman en raison de la répartition non normale des mesures. La droite de Tèissier (SCHERRER, 1984) a été choisie pour illustrer les relations biométriques (LT/PT, LT/DO) et l'égalité des pentes et des ordonnées à l'origine des différentes droites a été testée par un test $Z$. La variation du diamètre du nucleus dans les différents lots a été testée à l'aide d'une analyse de variance à un critère de classification (ANOVA). Les diamètres des LAC ont été comparés au moyen d'un test non paramétrique de Mann-Whitney (SCHERRER, 1984).

\section{RÉSULTATS}

\section{Croissance relative}

En Vilaine, les droites de Teissier illustrant les relations entre la taille et le poids des anguilles (fig. 1a) ou entre la taille des anguilles et le diamètre de leurs otolithes (fig. 2a) ne différaient pas significativement en fonction du secteur, estuarien ou fluvial $\left(Z<Z_{a 0,5}\right)$. Dans les deux cas, ces mesures étaient fortement corrélées $\left(r_{e}=0,90 ; r_{f}=0,72\right)$. Dans les échantillons capturés en Loire, la pente de la droite de Teissier ajustée aux tailles et aux poids des anguilles était significativement supérieure dans le lot estuarien $\left(Z>Z_{a 0,5} ;\right.$ fig. $\left.1 b\right)$. Les relations ente la taille des anguilles et le diamètre de leurs otolithes ne présentaient pas de différence significative entre les lots estuariens ou fluviaux $\left(Z<Z_{a 0,5}\right.$; fig. 2b) et les corrélations étaient significatives $\left(r_{e}=0,90 ; r_{f}=0,94\right)$.

\section{Patron des Lignes d'Arrêt de Croissance des otolithes}

La valeur moyenne du diamètre du nucleus était identique dans tous les lots : aucune différence significative n'a été observée ( $\mathrm{F}<\mathrm{Fa}$ dans tous les cas, Tableau I), et le coefficient de variation autour des mesures était faible (<10\%; Tableau I).

Pour une même classe de taille, les otolithes des anguilles fluviales présentaient jusqu'à deux fois plus de. LAC que ceux des lots estuariens (fig. 3 et 4). Cette différence du nombre maximum de LAC par otolithe a été observée à partir de la classe de taille 200$250 \mathrm{~mm}$ en Vilaine, à partir de la classe $250-300 \mathrm{~mm}$ en Loire. Dans le lot fluvial, le nombre de LAC n'était pas corrélé à la taille des anguilles $\left(r_{f}=0,37\right)$; la corrélation était forte dans le lot estuarien $\left(r_{e}=0,82\right)$. 

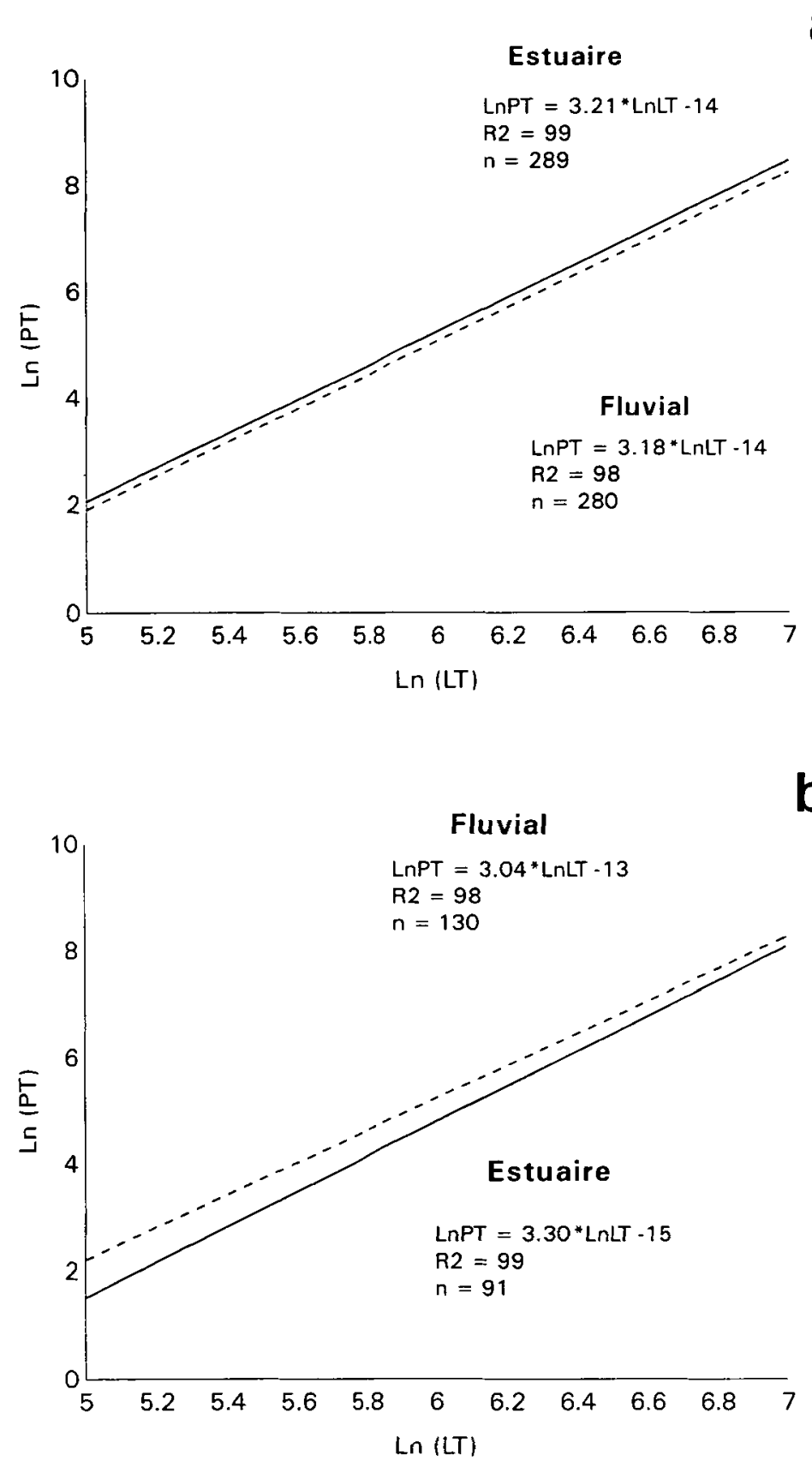

Figure 1 : Modèle de Teissier ajusté à la longueur et au poids des anguilles : a-Vilaine, b-Loire.

LnLT = logarithme népérien de la longeur totale

LnPT = logarithme népérien du poids total $\mathbf{n}=$ effectif

Figure 1 : Eel length-weight relationships fitted by Teissier models : a-Vilaine, b-Loire. LT = Neperian logarithm of total length PT $=$ Neperian logarithm of total weight n = sample size 

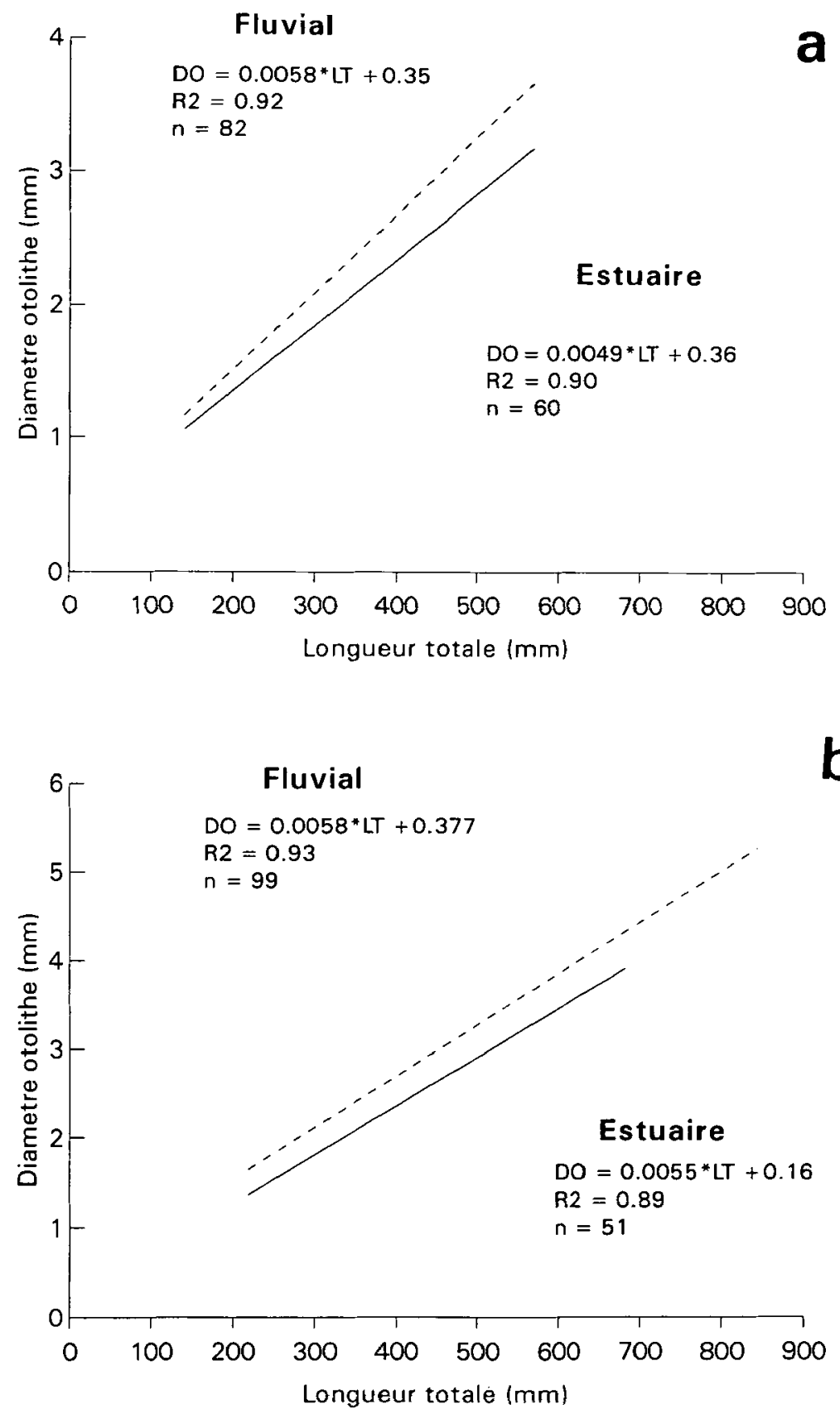

Figure 2 : Modèle de Teissier ajusté au diamètre des otolithes et à la longueur des anguilles :

a-Vilaine, b-Loire.

$\mathrm{DO}=$ diamètre des otolithes

LT = longueur totale

$\mathrm{n}=$ effectif

Figure 2 : Otolith diameter-eel length relationships fitted by Teissier models : a-Vilaine, b-Loire.

$\mathrm{DO}=$ otolith diameter

LT = total length

n = sample size 
Tableau I : Diamètre moyen du nucleus des otolithes d'anguilles.

DNS = différence non significative

Table I : Mean diameter of eel otolith nucleus.

DNS = non significant difference.

\begin{tabular}{lcccc}
\hline & Effectif & $\begin{array}{c}\text { Diamètre du } \\
\text { Nucleus } \mu \mathrm{m}\end{array}$ & $\begin{array}{c}\text { Coefficient de } \\
\text { Variation } \%\end{array}$ & Test de Fisher \\
\hline $\begin{array}{l}\text { Vilaine } \\
\text { Estuaire }\end{array}$ & 58 & 325 & 8 & F $=1.35$ \\
$\begin{array}{l}\text { Fluvial } \\
\text { Loire }\end{array}$ & 82 & 325 & 10 & DNS \\
Estuaire & 20 & 352 & 10 & F $=1.28$ \\
Fluvial & 14 & 354 & 6 & DNS \\
\hline
\end{tabular}

Tableau II : Fréquence d'observation des deux catégories de LAC dans les-otolithes d'anguilles.

$\mathbf{N}=$ effectif

$\mathbf{L T}=$ gamme des tailles

Fréq/échant $=$ fréquence d'observation au sein de l'échantillon

$\mathrm{Nb} /$ otolithe $=$ nombre maximal par otolithe

Table II : Frequency of both types of zones on eel otoliths.

$\mathrm{N}$ = sample size

$\mathbf{L T}=$ size range

Fréq/échant $=$ frequency of observation in a sample

$\mathrm{Nb} /$ otolithe $=$ maximal number by otolith

\begin{tabular}{lcccccc}
\hline & N & LT mm & Fréq/échant & Nb/otolithe & Fréq/échant & Nb/otolithe \\
\hline $\begin{array}{l}\text { Vilaine } \\
\text { Estuaire }\end{array}$ & 57 & $130-800$ & $5 \%$ & $<5$ & $100 \%$ & $<7$ \\
$\begin{array}{l}\text { Fluvial } \\
\text { Loire }\end{array}$ & 81 & $150-800$ & $100 \%$ & $>10$ & $100 \%$ & $<15$ \\
$\begin{array}{l}\text { Estuaire } \\
\text { Fluvial }\end{array}$ & 20 & $250-700$ & $8 \%$ & $<5$ & $100 \%$ & $<8$ \\
\hline
\end{tabular}



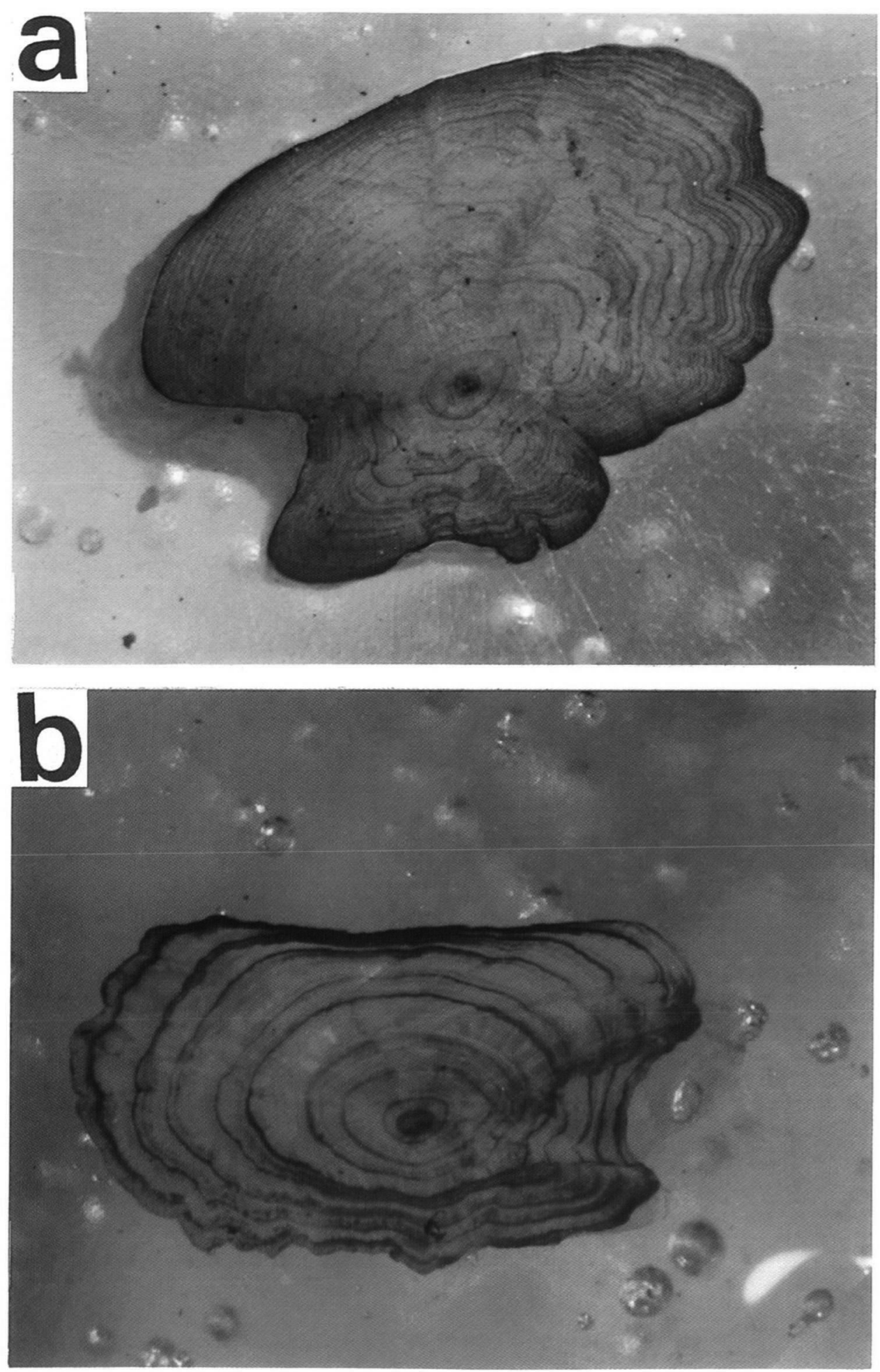

Figure 3 : Coloration des Lignes d'Arrêt de Croissance des otolithes d'anguilles au bleu de toluidine ( $x 50$ ) : a-anguille fluviale ( $L T=684 \mathrm{~mm}$ ), b-anguille estuarienne (LT $=644 \mathrm{~mm}$ ).

Figure 3 : Toluidine blue staining of growth rest zones on eel otoliths $(\times 50)$ : a-fluvial eel $($ LT $=684 \mathrm{~mm})$, b-estuarine eel $(L T=644 \mathrm{~mm})$. 


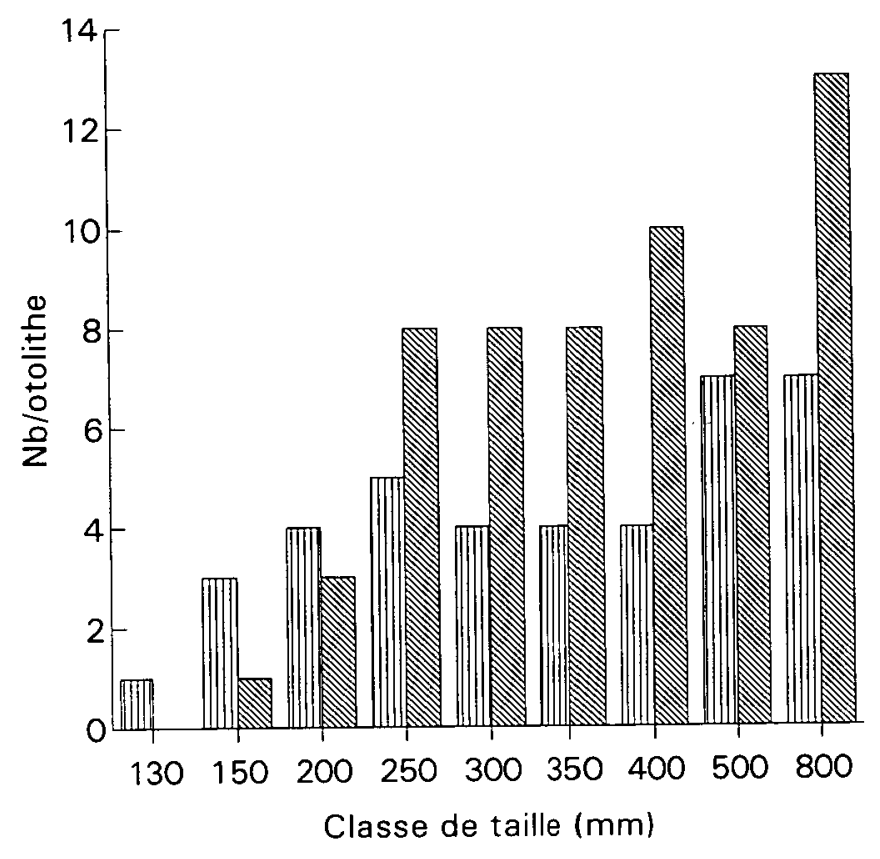

a

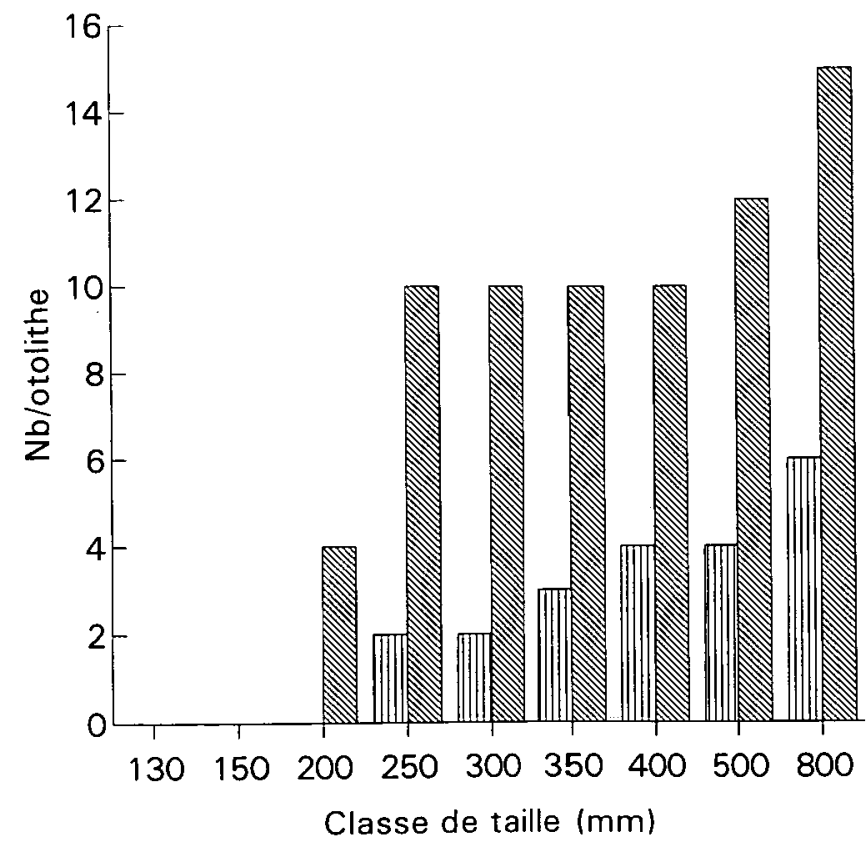

b

面 Estuaire

Fluvial

Figure 4 : Nombre de LAC (maximum) observées sur les otolithes selon la classe de taille des anguilles : a-Vilaine, b-Loire.

Figure 4 : Number (maximum) of growth rest zones observed on otoliths according to length class of eels : a-Vilaine, b-Loire. 


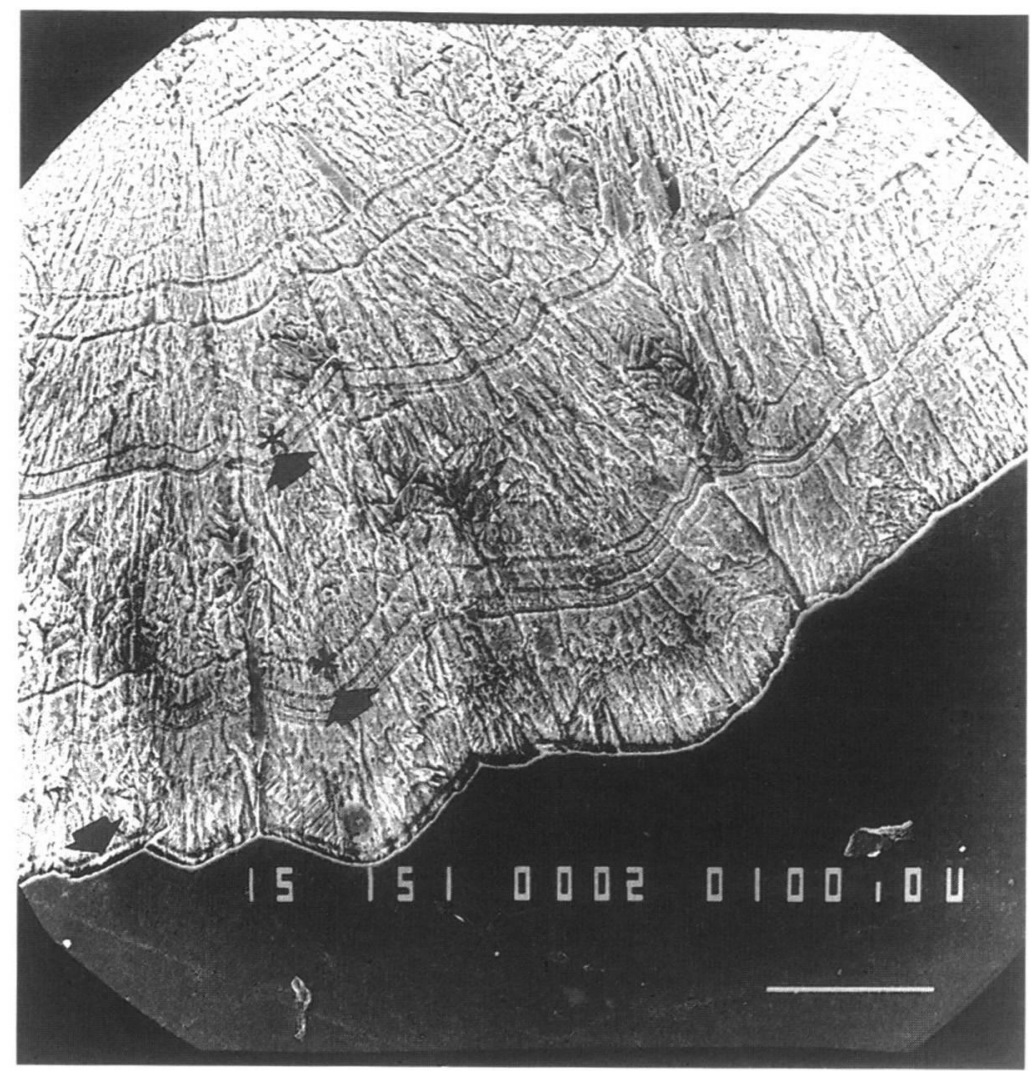

Figure 5 : Microstructure des Lignes d'Arrêt de Croissance des otolithes d'anguilles (x150).

= LAC composite annuelle

* = LAC simple surnuméraire

Figure 5 : Microstructure of growth rest zones of eel otoliths (x150).

- composite annual zone

* = simple supernumerary zone

\section{Microstructure des LAC}

Deux types de LAC ont été décrites en microscopie électronique à balayage (fig. 5) : des marques larges (> $10 \mu \mathrm{m}$ ) et composites, c'est-à-dire formées de plusieurs sillons, et des marques simples et plus étroites $(<5 \mu \mathrm{m})$, intercalées entre les marques composites. Les LAC composites ont été observées dans tous les otolithes (Tableau II). Les marques simples ont été observées chez tous les otolithes des anguilles fluviales $(100 \%$ des otolithes, en Vilaine comme en Loire) où elles étaient abondantes (+ de 10, Tableau II) ; dans les otolithes des lots estuariens, elles étaient peu abondantes (- de 5 ; Tableau II) et rarement observées : $5 \%$ des otolithes en Vilaine et $8 \%$ en Loire.

\section{Croissance des otolithes}

Une expérience de marquage à la calcéine, réalisée dans le bassin versant de la Vilaine, a permis de valider les LAC composites comme les marques annuelles hivernales (MOUNAIX, 1992). Leur agencement décrit la croissance de l'otolithe : compte tenu des effectifs réduits des échantillons et du cumul de plusieurs classes d'âge, il s'agit là d'une 




Figure 6 : Diamètre des 5 premières LAC annuelles, mesuré sur les otolithes des anguilles de la Vilaine.

Figure 6 : Diameter of the first 5 annual zones, measured in otoliths of eels from the Vilaine basin.

première approximation. Deux séquences ont été observées (fig. 6) : une première phase de croissance rapide (avant 3 ans), suivie d'une période de croissance ralentie (à partir de 4 ans). Le diamètre des trois premières marques annuelles était identique dans les deux secteurs, estuarien et fluvial (dans tous les cas, $Z<Z_{a 0,5}$ ). Au delà de la troisième marque, un retard de croissance apparaît sur les otolithes des anguilles fluviales $\left(Z>Z_{a 0,5}\right)$.

Aucune série caractéristique n'est apparue dans le patron d'agencement des marques simples, en dehors de la présence fréquente d'un groupe de LAC simples autour de la première marque composite de l'otolithe. En règle générale, les LAC simples étaient dispersées entre les LAC composites et leur agencement ne présentait aucune tendance identifiable.

\section{DISCUSSION}

Le patron de croissance des otolithes : un critère d'identification des anguilles estuariennes et fluviales.

Le patron des otolithes a permis de discriminer les anguilles selon leur habitat, estuarien ou fluvial, dans les deux bassins versants, la Loire et la Vilaine. Les critères d'identification sont le nombre et la microstructure des lignes d'arrêt de croissance. Ces 
critères s'ajustent à $95 \%$ des échantillons en Vilaine. Le barrage d'Arzal isole les deux secteurs du bassin versant et renforce les différences physico-chimiques entre les deux milieux (MARTIN; 1977). II représente un obstacle aux déplacements des anguilles (ELIE et RIGAUD, 1984) : le passage se fait épisodiquement à travers une écluse de navigation. La ségrégation est également forte en Loire (92\%) malgré l'absence de barrage estuarien. Ces observations indiquent la sédentarisation d'une partie des anguilles dans l'estuaire, comme déjà signalée par LABAR et al. (1987) et BERG (1989).

La distinction des deux sous-unités de la population d'anguilles est basée sur des différences de croissance. En effet, l'otolithe décrit les étapes de la croissance du poisson à travers sa structure et la séquence d'agencement des différentes couches (CAMPANA et NIELSON, 1985 ; CASSELMAN, 1990). Chez l'Anguille, les lignes d'arrêt de croissance larges et composites sont des marques annuelles (LECOMTE-FINIGER, 1992) déposées au printemps (MOUNAIX, 1992). Les marques simples correspondent aux "checks" non saisonniers, ou marques surnuméraires (LEE, 1982 ; BERG, 1985 ; LECOMTE-FINIGER, 1992) : elles résultent de perturbations de la croissance souvent liées à des modifications ponctuelles de l'environnement (LEE, 1982 ; CAMPANA et NIELSON, 1985) et peuvent être induites expérimentalement par choc thermique (BERGMAN et KARAKIRI, 1990). Décrites chez diverses espèces vivant en milieu marin, estuarien ou fluvial, elles ne semblent pas liées à la salinité mais peuvent correspondre à un choc halin.

Dans le cas des deux populations d'anguilles étudiées, l'abondance des LAC simples sur les otolithes fluviaux et leur rareté chez les otolithes estuariens indique des perturbations de la croissance plus nombreuses en milieu fluvial. La température est un facteur déterminant de la croissance des anguilles (BERG, 1985 et 1989) et l'activité métabolique est liée à des seuils thermiques (VOLLESTAD, 1989). La plus grande variabilité de la température du milieu fluvial est très probablement le facteur déterminant des fluctuations de la croissance des anguilles dans ce biotope ; par contraste, l'estuaire, par sa masse d'eau importante et son caractère généralement stratifié apparaît thermiquement plus stable, essentiellement régulé par les variations saisonnières du climat (MARTIN, 1977 ; BONNEFILE, 1980). Les conditions trophiques et le comportement alimentaire, tous deux variables selon le milieu (PAULOVITS et BIRÒ, 1986), sont également des facteurs déterminants de la croissance des anguilles. Par ailleurs, en Vilaine, la zone fluviale du bassin est caractérisée par une succession de biefs (ELIE et RIGAUD, 1984 ; LEGAULT et PORCHER, 1990), qui perturbent les déplacements des anguilles et influent ainsi sur leur croissance et leur abondance (APRAHAMIAN, 1988 ; LEGAULT, 1993). La synergie de ces facteurs, ainsi que les actions humaines sur le milieu (pollution, modification de l'habitat), entraînerait de fréquentes perturbations ponctuelles de la croissance des anguilles, inscrites sur l'otolithe sous la forme de marques simples. En estuaire, la croissance des anguilles serait essentiellement soumise au rythme saisonnier endogène : seules les marques annuelles sont enregistrées sur les otolithes.

Les critères biométriques (droites de Teissier et croissance relative des otolithes) ne sont pas discriminants. L'absence de différence statistique pourrait être liée au traitement global des données (toutes classes d'âge confondues). Par ailleurs, les deux sous-unités de la population ne sont peut-être pas suffisamment distantes (géographiquement et biologiquement) pour être identifiées par des critères biométriques.

Le pourcentage de non-discrimination (5\% en Vilaine et $8 \%$ en Loire) correspond à des anguilles pêchées en estuaire mais dont les otolithes comportaient des LAC simples. II pourrait s'agir de poissons vivant en zone fluviale aval, et prélevés lors d'un déplacement dans l'estuaire, ou traversant l'estuaire lors de la migration de dévalaison mais non identifiés comme argentés. Il peut aussi s'agir de poissons ayant subi des perturbations de la croissance, non liées aux caractéristiques climatiques du milieu.

\section{Vers une définition d'écotypes?}

Nos observations indiquent un fractionnement de la population d'anguilles en deux unités distinctes, estuarienne et fluviale, qui ne correspond pas aux théories de migrations continues ou séquencées d'anguilles (APRAHAMIAN, 1988 ; LEGAULT, com. pers., 1993 : 
observations de passes pièges en Bretagne). Dans les bassins versants français de la façade Atlantique, les observations de remontées massives de civelles ou de jeunes anguilles pigmentées attestent de la colonisation du bassin versant par les stades les plus jeunes. Dans le cas de petits bassins versants, il est probable que les migrations retardées (GUERAULT et al., 1992) aient une importance réduite, plus particulièrement en Vilaine : en effet, le barrage d'Arzal constitue un obstacle infranchissable en dehors des périodes d'ouverture des vannes. Il entraîne un regroupement des civelles en estuaire et conditionne très probablement les schémas de colonisation du bassin.

Deux phases de croissance de l'otolithe ont été observées : une période de croissance rapide jusqu'à trois ans puis un ralentissement de la croissance à partir de la quatrième année. Un modéle similaire de croissance en phases a été proposé pour l'anguille américaine (HANSEN et EVERSOLE, 1984 ; HELFMAN et al., 1984) et pourrait être liée aux modifications physiologiques résultant des processus de maturation (FERNANDEZ-DELGADO et al., 1989 ; VOLLESTAD, 1992). Ces hypothèses demandent à être testées sur un échantillon étendu, représentatif des structures de la population. Néanmoins, à travers ces indications, deux écotypes d'anguilles semblent pouvoir être définis dans le bassin versant de la Vilaine : un écotype fluvial où la croissance est perturbée et ralentie, un écotype estuarien où la croissance est régulière. La similitude des patrons des otolithes semble indiquer la validité de ces écotypes dans la population d'anguilles de la Loire malgré l'absence de barrage. De telles différences de croissance des anguilles à l'échelle d'un bassin versant ont été décrites par FERNANDEZ-DELGADO et al. (1989) dans le bassin du Guadalquivir (Espagne) ; HELFMAN et al. (1984) aboutissent aux mêmes conclusions pour la population d'anguilles américaines de la rivière Altamaha (Géorgie) : les anguilles estuairennes ont une croissance plus rapide. Les deux écotypes ainsi définis ne correspondent pas à des populations génétiquement distinctes, mais à des sous-unités écologiques.

Enfin, l'argenture, le déclenchement de la migration catadrome et la maturation sexuelle sont des processus physiologiques liés au taux de croissance de l'anguille (VOLLESTAD et JONSSON, 1986). De même, la détermination du sexe dépend des facteurs environnementaux et du taux de croissance (HELFMAN et al., 1987). Les différences de croissance entre les deux écotypes auraient donc des conséquences déterminantes sur les stratégies écologiques dans les différents compartiments d'un bassin versant, en particulier un cycle biologique plus long en milieu fluvial. Cette hypothèse recoupe les observations de TULONEN (1989) en Norvège et de FERNANDEZ-DELGADO et al. (1989) en Espagne, décrivant un départ plus précoce des anguilles vivant en milieu estuarien. La prise en compte de ces différentes stratégies est déterminante pour l'étude des populations d'anguilles et la gestion des stocks.

\section{CONCLUSION :}

\section{UN OUTIL POUR L'ÉTUDE ET LA GESTION DES STOCKS D'ANGUILLES ?}

Le patron d'agencement des structures de l'otolithe est caractéristique de l'habitat (estuarien ou fluvial) des anguilles : il décrit les différences de croissance dans ces deux biotopes, et permet d'identifier a posteriori le milieu de sédentarisation des anguilles. Les critères à analyser sont : le nombre de LAC, l'aspect de leur agencement (régulier ou feuilleté) et leur microstructure. Dans nos échantillons, le pourcentage d'erreur de ces critères était faible $(<8 \%)$. Cet outil présente l'avantage, par rapport aux méthodes biométriques d'identification des stocks, de permettre l'identification individuelle du poisson. II rend ainsi possible la quantification du taux de mélange d'une population.

Du point de vue de l'étude des populations d'anguilles à l'échelle du bassin versant, l'analyse du patron des otolithes autorise une approche nouvelle dans l'exploration des stratégies migratoires : il permet d'étudier la colonisation des zones amont à partir des otolithes des anguilles de dévalaison (anguilles argentées). Ce stade faisant l'objet d'une exploitation commerciale, il est possible d'échantillonner au sein des captures pour évaluer la part fluviale des individus migrateurs et tenir compte des deux stocks d'anguilles pour l'aménagement des bassins versants (passes, etc...) et la gestion des pêcheries d'anguilles. 


\section{REMERCIEMENTS}

Cette étude a été financée par la Direction Générale de l'Enseignement et de la Recherche du Ministère de l'Agriculture et de le Forêt et a fait l'objet d'une allocation de recherche attribuée par le Ministère de la Recherche et de la Technologie.

\section{BIBLIOGRAPHIE}

APRAHAMIAN M.W., 1988. Age structure of eel, Anguilla anguilla (L.) populations in the river Severn, England, and in the river Dee, Wales. Aquacult. Fish. Manage., 19, 365-376.

BAGLINIERE J.L., CASTANET J., CONAND F., MEUNIER F.J., 1992. Terminologie en sclérochronologie chez les vertébrés in BAGLINIERE J.L., CASTANET J., CONAND F., MEUNIER F. ed., Tissus durs et âge individuel des vertébrés, ORSTOM-INRA, Bondy, 443-447.

BERG R., 1985. Age determination of eels, Anguilla anguilla (L.) : comparison of field data with otolith ring patterns. J. Fish Biol., 26, 537-544.

BERG R., 1989. The growth of eels : a critical assessment of data from open waters. EIFAC, Working Party on Eel, Porto (Portugal), 11 p., ronéo.

BERGMAN R., KARAKIRI M., 1990. Experimental induction of biological tags in otoliths of 0-group plaice Pleuronectes platessa by starvation, temperature, and UV-B radiation. Mar. Ecol. Prog. Seri., 67, 227-233.

BONNEFILE R., 1980. Les phénomènes résiduels en estuaire. Océanis, 6 (4), 343-357.

CAMPANA S.E., NEILSON J.D., 1985. Microstructure of fish otoliths. Can. J. Fish. Aquat. Sci., 42, 1014-1032.

CASSELMAN J.M., 1990. Growth and relative size of calcified structure of fish. Trans. Am. Fish. Soc., 119, 673-688.

ELIE P., RIGAUD C., 1984. Etude de la population d'anguilles de l'estuaire et du bassin versant de la Vilaine : pêche - biologie - écologie. Examen particulier de l'impact du barrage d'Arzal. Rapp. CEMAGREF, $174 \mathrm{p}$.

FERNANDEZ-DELGADO C., HERNANDO J.A., HERRERA M., BELLIDO M., 1989. Age and growth of eel (Anguilla anguilla) in the estuary of the Guadalquivir River (South West Spain). J. Fish Biol., 34, 561-570.

GASCUEL D., 1987. La civelle d'Anguille dans l'estuaire de la Sèvre Niortaise : biologie, écologie, exploitation. Les Publications du Département Halieutique, 4 (1), 330p. + annexes.

GASCUEL D., FONTENELLE G., 1989. Caractéristiques biologiques d'un peuplement estuarien d'anguilles subadultes (estuaire de la Sèvre Niortaise, France). EIFAC, Working Group on Eel, Porto (Portugal), 12 p., ronéo.

GUERAULT D., LECOMTE-FINIGER R., DESAUNAY Y., BIAGIANTI-RISBOURG S., BEILLOIS P., GRELLIER P., 1992. Glass eel arrivals in the Vilaine estuary (Northern Bay of Biscay) in 1990: demographic features and early life history. Iri. Fish. Invest. (A Freshwat.), 36, 5-14.

HANSEN R.A., EVERSOLE A.G., 1984. Age, growth and sex-ratio of American eels in brackish water portions of South Carolina river. Trans. Am. Fish. Soc. 113, 744-749.

HARO A.J., KRUEGER W.H., 1991. Pigmentation, otolith rings, and upstream migration of juvenile American eels (Anguilla rostrata) in a coastal Rhode Island stream. Can. J. Zool,, 69 (3), 812-814.

HELFMAN G.S., BOZEMAN E.L., BROTHERS E.B., 1984 : Size, age and sex of American eels in a Georgia river. Trans. Am. Fish. Soc., 113, 132-141. 
HELFMAN G.S., FACEY D.E., HALES F.L.Jr., BOZEMAN E.L., 1987. Reproductive ecology of the American eel. Am. Fish. Soc. Symposium, 1, 42-46.

LABAR G.W., HERNANDO-CASAL J.A., FERNANDEZ-DELGADO C., 1987. Local movements and population size of European eels, Anguilla anguilla, in a small lake in southwestern Spain. Env. Biol. Fish., 19, 111-117.

LECOMTE-FINIGER R., 1992. Growth history and age at recruitment of the European glass eels (Anguilla anguilla) as revealed by otolith microstructure. Mar. Biol., 114, 205-210.

LEE T.W., 1982. Ageing and growth of eel population, Anguilla anguilla, in the lagoons of Arcachon Bay (France). J. Oceanol. Soc. Korea, 17 (2), 83-91.

LEGAULT A., PORCHER J.P., 1990. Distribution du peuplement d'anguilles à l'échelle régionale «Bretagne Basse-Normandie». Int. Revue ges. Hydrobiol., 75 (6), 794.

LEGAULT A., 1993. L'anguille : aménagement des obstacles à la migration. Publ. Assoc. Dév. Aquacult., 37, $33 \mathrm{p}$.

MARTIN J., 1977. Le milieu estuarien. La Recherche, 78 (8), 425-434.

MOUNAIX B., 1992. Intercalibration et validation des méthodes d'estimation de l'âge de l'Anguille européenne (Anguilla anguilla L.). Application au bassin versant de la Vilaine, Bretagne. Les Publications du Département Halieutique, 14, $146 \mathrm{p}$.

PAULOVITS G., BIRÒ P., 1986. Age determination and growth of eel, Anguilla anguilla (L.), in Lake Fertö, Hungary. Fish. Res., 4, 101-110.

SCHERRER B., 1984. Biostatistique. Gaëtan Morin ed., Chicoutimi Québec, 850 p.

TULONEN J., 1989. Growth and sex-ratio of eels (Anguilla anguilla) of known age in four small lakes in southern Finland. EIFAC, Working Party on Eel, Porto (Portugal), 20 p., ronéo.

VOLLESTAD L.A., 1989. Age determination and growh of yellow eels, Anguilla anguilla (L.), from brackish water, Norway. J. Fish Biol., 26, 521-525.

VOLLESTAD L.A., 1992. Geographic variation in age and length at metamorphosis of maturing European eel: environmental effects and phenotypic plasticity. J. Anim. Ecol., 61, 41-48.

VOLLESTAD L.A., JONSSON, B., 1986. Life-history characteristics of the European eel (Anguilla anguilla) in the Imsa River, Norway. Trans. Am. Fish.Soc., 115, 864-871 